\title{
Resonant microwave cavity for 8.5-12 GHz optically detected electron spin resonance with simultaneous nuclear magnetic resonance
}

John S. Colton

john_colton@byu.edu

L. R. Wienkes

Follow this and additional works at: https://scholarsarchive.byu.edu/facpub

Part of the Astrophysics and Astronomy Commons, and the Physics Commons

\section{Original Publication Citation}

Resonant microwave cavity for $8.5-12 \mathrm{GHz}$ optically-detected electron spin resonance with simultaneous nuclear magnetic resonance, J.S. Colton, L.R. Wienkes* , Rev. Sci. Inst. 8, 3516 (29). The original version may be found at: http://rsi.aip.org/resource/1/rsinak/v8/i3/p3516_s1

\section{BYU ScholarsArchive Citation}

Colton, John S. and Wienkes, L. R., "Resonant microwave cavity for 8.5-12 GHz optically detected electron spin resonance with simultaneous nuclear magnetic resonance" (2009). Faculty Publications. 140.

https://scholarsarchive.byu.edu/facpub/140

This Peer-Reviewed Article is brought to you for free and open access by BYU ScholarsArchive. It has been accepted for inclusion in Faculty Publications by an authorized administrator of BYU ScholarsArchive. For more information, please contact ellen_amatangelo@byu.edu. 


\section{AIP Scientific Instruments}

\section{Resonant microwave cavity for $8.5-12 \mathrm{GHz}$ optically detected electron spin resonance with simultaneous nuclear magnetic resonance}

\section{J. S. Colton and L. R. Wienkes}

Citation: Rev. Sci. Instrum. 80, 035106 (2009); doi: 10.1063/1.3095683

View online: http://dx.doi.org/10.1063/1.3095683

View Table of Contents: http://rsi.aip.org/resource/1/RSINAK/v80/i3

Published by the American Institute of Physics.

\section{Related Articles}

Coherent Josephson phase qubit with a single crystal silicon capacitor Appl. Phys. Lett. 102, 012602 (2013)

A rapid high power microwave switch based on multipactor electrons flow Appl. Phys. Lett. 102, 013504 (2013)

Design and development of $3 \mathrm{~dB}$ patch compensated tandem hybrid coupler Rev. Sci. Instrum. 84, 014702 (2013)

Terahertz filters based on frequency selective surfaces for high-speed terahertz switch J. Appl. Phys. 113, 014504 (2013)

Germanium metal-semiconductor-metal photodetectors evanescently coupled with upper-level silicon oxynitride dielectric waveguides

Appl. Phys. Lett. 101, 261109 (2012)

\section{Additional information on Rev. Sci. Instrum.}

Journal Homepage: http://rsi.aip.org

Journal Information: http://rsi.aip.org/about/about_the_journal

Top downloads: http://rsi.aip.org/features/most_downloaded

Information for Authors: http://rsi.aip.org/authors

\section{ADVERTISEMENT}

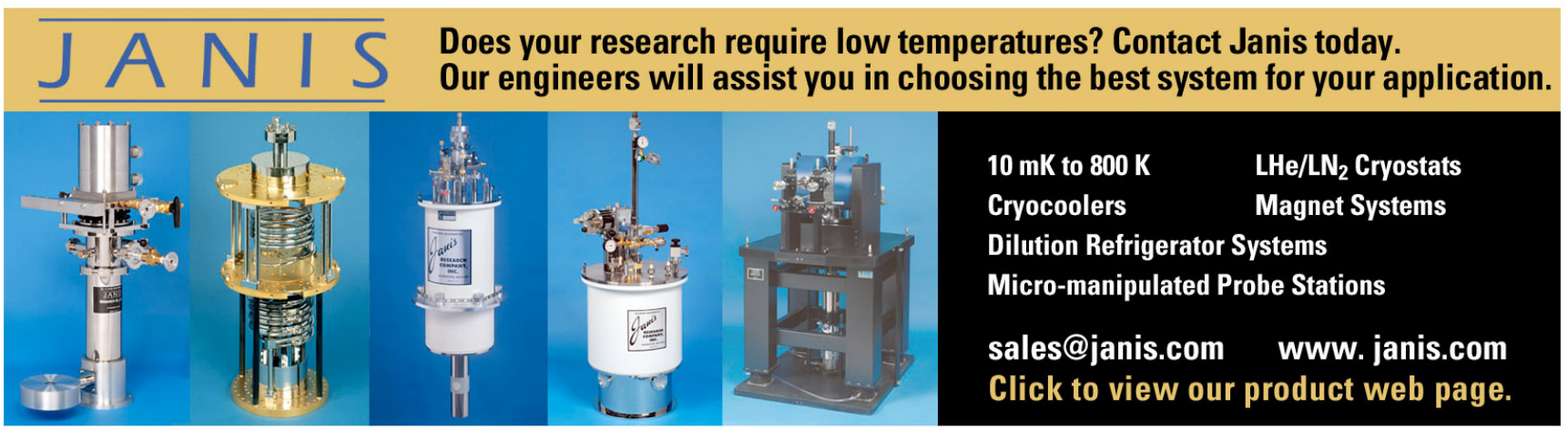




\title{
Resonant microwave cavity for $8.5-12 \mathrm{GHz}$ optically detected electron spin resonance with simultaneous nuclear magnetic resonance
}

\author{
J. S. Colton ${ }^{1, a)}$ and L. R. Wienkes ${ }^{2}$ \\ ${ }^{1}$ Department of Physics and Astronomy, Brigham Young University, Provo Utah 84602, USA \\ ${ }^{2}$ School of Physics and Astronomy, University of Minnesota, Minneapolis, Minnesota 55455, USA
}

(Received 10 July 2008; accepted 16 February 2009; published online 16 March 2009)

\begin{abstract}
We present a newly developed microwave resonant cavity for use in optically detected magnetic resonance (ODMR) experiments. The cylindrical quasi-TE $\mathrm{T}_{011}$ mode cavity is designed to fit in a 1 in. magnet bore to allow the sample to be optically accessed and to have an adjustable resonant frequency between 8.5 and $12 \mathrm{GHz}$. The cavity uses cylinders of high dielectric material, so-called "dielectric resonators," in a double-stacked configuration to determine the resonant frequency. Wires in a pseudo-Helmholtz configuration are incorporated into the cavity to provide frequencies for simultaneous nuclear magnetic resonance (NMR). The system was tested by measuring cavity absorption as microwave frequencies were swept, by performing ODMR on a zinc-doped InP sample, and by performing optically detected NMR on a GaAs sample. The results confirm the suitability of the cavity for ODMR with simultaneous NMR. () 2009 American Institute of Physics. [DOI: 10.1063/1.3095683]
\end{abstract}

\section{INTRODUCTION}

Spin states of electrons in semiconductors are wellknown candidates for "qubits" to be used in quantum computing schemes. ${ }^{1}$ In order for a quantum computer to work, it must be able to do many operations before the quantum states comprising the qubits experience substantial decoherence. Electron spin resonance (ESR), sometimes called electron paramagnetic resonance, is a well-established technique for studying spin-related properties of electrons inside solids ${ }^{2}$ and is a natural way of probing spin states for their suitability in quantum computing. Of the various spin lifetimes that can be measured, the most important one from a quantum computing perspective is the $T_{2}$ spin lifetime, also called the "transverse lifetime" or "spin dephasing time."

The $T_{2}$ lifetime can be directly probed by the spin echo technique, a resonance technique which uses microwave pulses to coherently rotate spins between states in order to eliminate inhomogeneous dephasing. ${ }^{3}$ In spin echo experiments, a resonant cavity is typically used to increase the resonant oscillating magnetic field at the sample in order to make the controlling pulses as short as reasonably possible. We therefore wanted to design a resonant cavity which could be used for spin echo-type experiments in semiconductors. We were particularly interested in constructing a cavity for GaAs and related materials, since although GaAs is a popular semiconductor with known long spin lifetimes and is often studied for quantum computing applications, ${ }^{4}$ spin echo experiments in GaAs have as yet been unsuccessful. Such experiments in GaAs are challenging due (in large part) to the substantial electron-nuclear interaction in that material, but experiments are in progress using the resonant cavity described below.

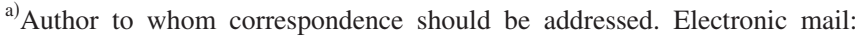
john_colton@byu.edu. Formerly at University of Wisconsin-La Crosse.
}

\section{DESIGN CONSIDERATIONS}

Our first design choice was for the cavity to have a resonant frequency around $10 \mathrm{GHz}$. For GaAs, with an electron spin $g$-factor of $|g| \approx 0.44,{ }^{5}$ the resonance equation

$$
h f=g \mu_{B} B,
$$

predicts the resonant field corresponding to $10 \mathrm{GHz}$ to be $1.62 \mathrm{~T}$. This field is desirable for prospective GaAs experiments for several reasons. First, the longitudinal lifetime, $T_{1}$, in GaAs is known to depend strongly on magnetic field. ${ }^{6-8}$ Given the field dependence of $T_{1}$, and the expectation that $T_{2}$ should be of the order of $T_{1}$, , this choice of magnetic field should be in the right regime to allow $T_{2}$ lifetimes of the order of microseconds to be observed. Second, a resonant frequency around $10 \mathrm{GHz}$ makes it possible to probe GaAs quantum well structures, which can have $|g|$ substantially lower than $0.44,{ }^{10}$ even given the practical $7-8 \mathrm{~T}$ field limit on many popular optically accessible superconducting magnet systems (such as our own). Third, $10 \mathrm{GHz}$ places the microwave frequencies solidly in the middle of the $X$-band, which is well known and often used in the microwave world. Specifically, it is low enough in frequency that coaxial cables may be used instead of waveguide and relatively inexpensive sources and amplifiers may be obtained.

Given the choice of a $\sim 10 \mathrm{GHz}$ resonant cavity, the second design consideration was for the cavity to fit inside our 1 in. magnet bore: the diameter of the cavity must be a bit under an inch. However, a 1 in. or smaller diameter traditional hollow metal cavity would have a much higher resonant frequency. Therefore, we substantially increased the optical path length inside the resonant cavity by adding high dielectric material; see Sec. III technical description below.

The third factor to substantially influence our design is the desire to do optically detected experiments. Quantum computing devices will eventually need to use individual 
qubits. Such a small quantity of spins cannot be probed by traditional ESR methods. Optical methods, on the other hand, have been shown to be able to address substantially less amount of material than traditional absorption ESR, ${ }^{11}$ in some cases even individual spins. ${ }^{12}$ Optical detection may also offer an advantage over traditional ESR, in that all of the signal is coming through direct (optical) interactions with the sample and not from, e.g., stray signal from paramagnetic impurities inside dielectrics in the microwave cavity. GaAs has previously been studied extensively through optical means, using techniques such as optical orientation/detection of spins ${ }^{13}$ and time-resolved Faraday/Kerr rotation. ${ }^{14}$ The desire for optical access to the sample leads to using the cylindrical $\mathrm{TE}_{011}$ mode. This mode is commonly used for optical experiments since the surface currents allow holes to be made in the side of the cavity without substantial degradation of cavity quality. ${ }^{15}$ This mode also provides a large $B$-field and a small $E$-field at the center of the cavity where the sample is placed, precisely what is desired for magnetic resonance. To obtain the optical access, we use two separate cylinders of the high dielectric material, separated by a small distance, with the sample placed between them. All the experiments presented below, as well as the ongoing spin echo experiments referred to above, are done through optical detection schemes.

Fourth, in the cavity designed for optically detected electron resonance, we must also be able to simultaneously perform nuclear magnetic resonance (NMR). This is extremely important in quantum-dot and $n$-doped GaAs where localized electrons interact strongly with the nuclei, all isotopes of which have a net spin; for example, previous experiments found that ESR could not be observed unless the nuclei were simultaneously resonated. ${ }^{16,17}$

Finally, as a less important item, since the spin lifetimes depend on magnetic field, when studying similar samples with slightly different $g$-factors (e.g., due to a slightly different quantum well widths or a slightly different chemical composition), it may be advantageous to have a range of cavity resonant frequencies. This would potentially allow experimenters to compare the different samples' spin properties at similar magnetic fields. The final design consideration was therefore for the cavity to allow a number of resonant frequencies; our design uses interchangeable dielectrics to accomplish this (see below).

\section{TECHNICAL DESCRIPTION}

As mentioned above, the design parameters of a 1 in. bore and a $10 \mathrm{GHz}$ resonant frequency lead to using a dielectric material inside the cavity in order to substantially increase the optical path length. Modern dielectrics designed for such a purpose are known as "dielectric resonators" (DRs) and are sold by companies such as Trans-Tech (Adamstown, MD), Murata (Smyrna, GA), and Emerson \& Cuming (Randolph, MA). We used two different types of cylindrical DRs in our resonant cavity, manufactured by Trans-Tech and Murata. The Trans-Tech DRs have $\varepsilon=14$ and an outer diameter (OD) of $10 \mathrm{~mm}$, while the Murata DRs have $\varepsilon=29.7$ and an OD of $5.5 \mathrm{~mm}$.

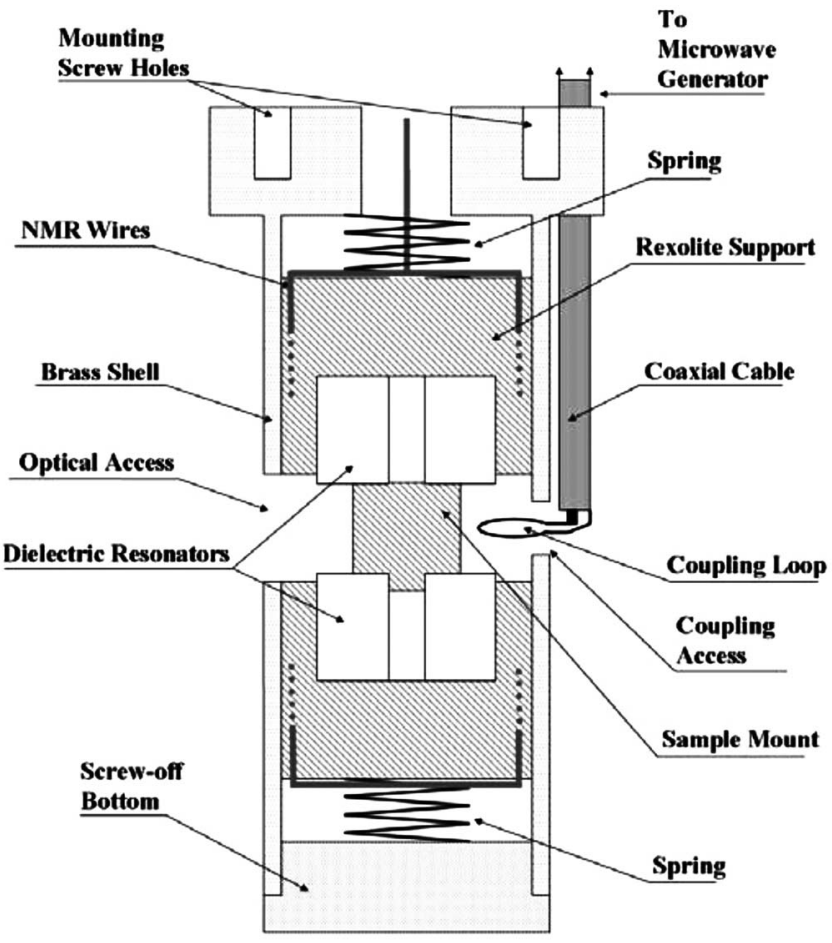

FIG. 1. Cross-sectional view of the resonant cavity structure. The sample was mounted on the left-hand side (as oriented in the figure), facing the optical access window. The sample mount contained a recess (not shown) so that the sample was at the exact center of the cavity.

The use of high dielectric materials as resonators is not new; the concept was pioneered by Richtmyer in 1939, who discussed bending a dielectric waveguide into a ring structure. ${ }^{18}$ Since then, DRs have become common in many applications, including use as microwave cavities for ESR. ${ }^{19}$

For much of our own design, we followed the example of Sienkiewicz et al.: $:^{20,21}$ (1) we used a double-stacked DR configuration with the sample placed between two identical, vertically stacked, DRs, (2) we used Rexolite (a material with a small loss tangent at microwave frequencies, made by C-LEC Plastics, Beverly, NJ) as a spacer material to support the DRs and the sample in their proper positions, and (3) we used a horizontal loop half-way down the cavity to couple the microwaves into the cavity and "ring" the cavity in the proper $\mathrm{TE}_{011}$ mode. See Fig. 1 .

However, whereas Sienkiewicz used a double-stacked DR configuration to provide frequency tuning in a resonator for stopped-flow free-radical experiments ${ }^{20}$ and to provide space for a sapphire-anvil cell in a resonator for highpressure experiments, ${ }^{21}$ we employed the double-stacked configuration to provide direct optical access to the sample in our cavity resonator. This contrasts with the previous optical DR-based microwave cavities constructed by Schreiner et al. ${ }^{22,23}$ to study AlGaAs/GaAs heterostructures using optical excitation and detection and Stavitski et al. ${ }^{24}$ and Blank et $a .^{25}$ to study molecular radical-triplet pair interactions using a laser to illuminate the sample but using traditional microwave absorption to detect the signal. Schreiner et al. and Stavitski et al. both used a single DR with a hole drilled from the outside into the middle where the sample was placed. Blank et al. considered that approach, but rejected it in favor 
of a transparent DR because they found the hole-in-DR approach significantly decreased the $Q$ of the resonator and distorted its resonant mode. We similarly dismissed the idea of a hole bored into a single DR because our simulations show that a hole in the DR substantially distorts the symmetry of the $\mathrm{TE}_{011}$ field inside the cavity. Blank et al. considered a double-stacked DR configuration like the one we used, but decided against it in favor of their transparent DR approach because the oscillating magnetic field in the middle of two stacked DRs is weaker than the field at the center of a single DR. On the other hand, if a slightly weaker field is allowable, a dual-stacked DR system allows the use of commercial, nontransparent DRs as opposed to the more specialized transparent DR used by Blank et al. (ferroelectric $\mathrm{KTaO}_{3}$ ). In the double-stacked approach the sample is also more directly accessible to light (e.g., for optical detection of the ESR signal) than when the sample is behind a high dielectric material.

In our double-stacked configuration, two identical cylindrical DRs are stacked on top of each other, separated by $5 \mathrm{~mm}$ via a sample mount made of Rexolite. The sample mount is centered in the $x-y$ plane by fitting tightly with the lower DR-a $2 \mathrm{~mm}$ diameter knob extends downward from the sample mount into a corresponding hole in the lower DR. The hole in the DR extends completely through the DR along the cylindrical axis. For the sake of symmetry, the upper DR also has a hole on its axis. The Trans-Tech DRs are thus $10 \mathrm{~mm}$ OD, $2 \mathrm{~mm}$ inner diameter (ID), with heights

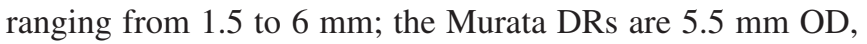
$2 \mathrm{~mm}$ ID, and have a $2.44 \mathrm{~mm}$ height.

The sample is placed on its mount at the midpoint between the two DRs. The sample mount, DRs, and Rexolite support structures are all placed inside a brass cylindrical shell. The brass shell has approximately $25 \mu \mathrm{m}$ of silver electroplated on it to improve conductivity and has a thin gold coating to protect the silver. The cavity is approximately $46 \mathrm{~mm}$ long with a $16 \mathrm{~mm}$ OD. The OD expands to $22 \mathrm{~mm}$ at the top so it can be mounted on a hanging support. As verified by simulations (see below), the microwave fields are mainly confined to regions near the DRs; therefore the dimensions of the brass cavity only affect the resonant frequency in a secondary manner. Or, as another way of looking at it, the total optical path length does not change by a substantial amount if the brass dimensions are varied slightly. A $6 \mathrm{~mm}$ diameter hole in the brass shell halfway down the $z$-axis (on the left in Fig. 1) provides the optical access.

We can easily switch DR configurations by using slightly different Rexolite holders, see Fig. 2. Since the resonant frequency of the cavity is primarily set by the DRs, interchanging the DRs in this fashion allows us to obtain a variety of resonant frequencies using the same brass cavity shell. The resonant frequencies obtained this way range from 8.5 to $12 \mathrm{GHz}$; details are provided in Table I. Our method of tuning the cavity's resonant frequency contrasts with that of Sienkiewicz et al., who designed resonant cavities which could change frequencies continuously in a $\sim 1-2 \mathrm{GHz}$ range by changing distances between DRs (Ref. 20) or by changing the total cavity height. ${ }^{26}$

The Rexolite pieces supporting the DRs are held in place

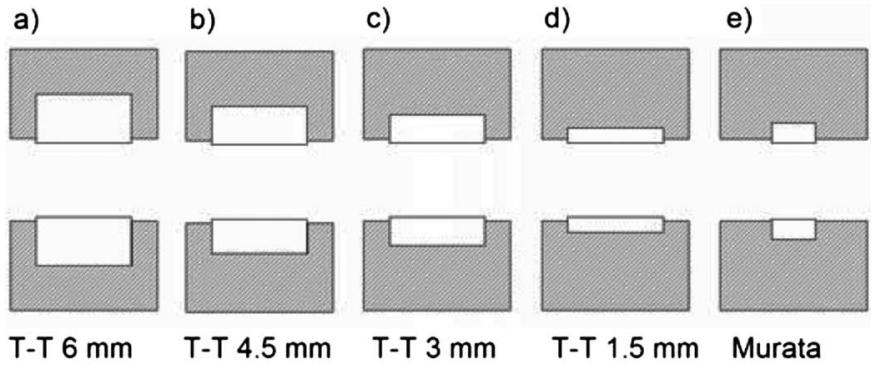

FIG. 2. Different DR configurations. The shaded area is the Rexolite support structure, while the white area is the DR. The sample mount (not shown) would be placed between the upper and lower halves. Configurations (a)-(d) are for Trans-Tech DRs of various thicknesses; thickness listed is that of a single DR. Configuration (e) is for the Murata DR.

and centered vertically by nonmagnetic brass springs at the top and bottom. Since the fields are small at the top and bottom ends of the cavity, these metallic springs did not substantially affect either the resonant frequency or the quality factor ( $Q$-factor) of a given cavity configuration. To transport the microwaves down to the cavity, we use a 0.141 in. semirigid coaxial cable; its inner conductor is bent around to form the coupling loop. The coupling loop has its own hole $(3 \mathrm{~mm}$ diameter) for access into the cavity, $180^{\circ}$ away from the hole for optical access. The loop is positioned halfway down the cavity and extends a few millimeters into the cavity proper. Before placing the cavity inside the magnet, we adjust the position and angle of the coupling loop manually until critical coupling is achieved; there are no mechanisms to alter the coupling once the cavity is inside the magnet bore.

Wires for performing NMR are introduced into the cavity via a $5 \mathrm{~mm}$ diameter hole at the top of the brass shell. The wires go into the cavity and then are wrapped around the sample. To minimize loss, in regions of high electric field, the wires travel perpendicularly to the electric field lines. Since the electric field lines in the $\mathrm{TE}_{011}$ mode run azimuthally, we put the NMR wires in the cavity in a pseudoHelmholtz geometry. ${ }^{27}$ This is similar to other cavities designed for electron-nuclear double resonance. ${ }^{26,28}$ The NMR wires run in a rectangular loop: vertically past the sample through the main part of the cavity and horizontally at the ends of the cavity where the electric field is weak. Holes and/or slots in the Rexolite supports provide a guide for the

TABLE I. Cavity resonant frequencies and critically coupled $Q$-factors for $300 \mathrm{~K}$ operation with no sample inside the structure. Addition of a small GaAs sample typically shifts the resonance down by $\sim 60 \mathrm{MHz}$ and slightly decreases the $Q$-factor. Operating at $1.5 \mathrm{~K}$ with liquid helium in the cavity typically increases the $Q$-factor slightly and shifts the resonance by a few tens of megahertz (this can be either up or down; the decrease in $f_{0}$ from the dielectric constant of liquid helium in the cavity competes with the increase in $f_{0}$ from thermal contraction).

\begin{tabular}{lccc}
\hline \hline DR configuration & $\begin{array}{c}\text { Predicted } f_{0} \\
(\mathrm{GHz})\end{array}$ & $\begin{array}{c}\text { Measured } f_{0} \\
(\mathrm{GHz})\end{array}$ & Measured $Q$ \\
\hline $2 \times 1.5 \mathrm{~mm}$ Trans-Tech & 11.76 & 11.9139 & 1900 \\
$2 \times$ MuRata & 10.85 & 10.7485 & 5000 \\
$2 \times 3.0 \mathrm{~mm}$ Trans-Tech & 9.898 & 9.8223 & 2500 \\
$2 \times 4.5 \mathrm{~mm}$ Trans-Tech & 9.072 & 9.0010 & 3100 \\
$2 \times 6.0 \mathrm{~mm}$ Trans-Tech & 8.63 & 8.5187 & 2500 \\
\hline \hline
\end{tabular}


NMR wires going through the cavity. In order to change DR configurations while the NMR wires are employed, the wires must be loosened or temporarily removed.

\section{CHARACTERISTICS OF THE RESONANT CAVITY: THEORY AND SIMULATION}

The label on a TE mode specifies the $z$-component of the magnetic field; in our case, the $\mathrm{TE}_{011}$ mode $B_{z}$ has no $\phi$ dependence, one antinode across the center, and one antinode top to bottom. ${ }^{29}$ For a hollow cylindrical conducting shell, all components of the fields can be obtained analytically. As stated earlier, this mode has a large magnetic field and a small electric field at the position of the sample. The resonant frequencies can be obtained via the general equation for $\mathrm{TE}_{m n p}$ modes:

$$
\left(\frac{2 \pi f_{\text {res }, m n p}}{c}\right)^{2}=\left(\frac{x_{m n}}{a}\right)^{2}+\left(\frac{p \pi}{h}\right)^{2},
$$

where $m, n$, and $p$ are numbers describing the mode (e.g., 0 , 1 , and 1), $a$ is the cylindrical radius, $h$ is the height, and $x_{m n}$ is the $n$th root of $J_{m}^{\prime}$ (the first derivative of the $m$ th Bessel function).

When DRs are added into the cavity, the fields change compared to the fields in a pure $\mathrm{TE}_{011}$ mode. However, many aspects remain the same, such as small currents in the walls of the cylinder and a large $B$-field/small $E$-field at the center, hence, the terms "quasi- $\mathrm{TE}_{011}$ " mode or " $\mathrm{TE}_{01} \delta$ " mode are sometimes used for this case. ${ }^{30}$ Although an approximate formula for predicting the quasi- $\mathrm{TE}_{011}$ resonance of an isolated DR is given by Kaifez and Guillon ${ }^{30}$ and some formulas for predicting resonant frequencies for single and stacked DR configurations are given by Jaworski et al. ${ }^{31}$ these do not match our observed resonant frequencies very well. Instead, we employed numerical simulations to obtain the resonant frequencies of our complicated structures.

To simulate our design and predict resonant frequencies for new configurations, we used the "Eigenmode solver" module of the CST MICROWAVE STUDIO computer program. The three-dimensional solver allowed us to visually inspect each resonant mode in order to find the mode whose field pattern was $\mathrm{TE}_{011}$-like and then obtain a prediction for the mode's frequency. These predictions were later compared to the experimentally measured resonant frequencies and were found to be very accurate (see Table I). The measured and predicted resonant frequencies are within $1.4 \%$ of each other for all cavity configurations. We suspect the small discrepancy is primarily due to differences between the actual cavity configurations and the models used in our simulations; we simplified the models somewhat in order to speed up simulations. Figure 3 shows the magnetic field close to the center of the cavity for one particular DR configuration.

\section{CHARACTERISTICS OF THE RESONANT CAVITY- EXPERIMENT}

\section{A. Resonant frequencies}

To measure the resonant frequency of a given configuration, we produced microwaves using an Agilent E8257D synthesizer and shined them into the cavity via coaxial cable. (a)
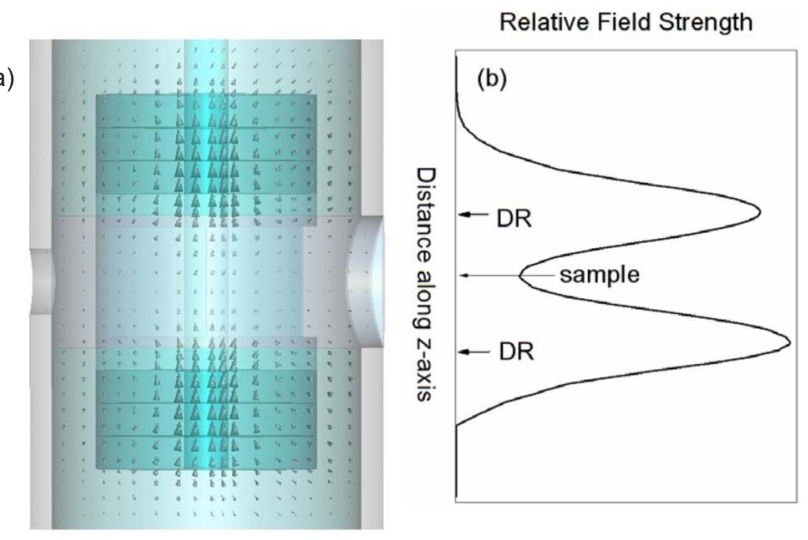

FIG. 3. (Color online) Magnetic field inside the resonant cavity for the 4.5 $\mathrm{mm}$ Trans-Tech configuration from the CST MICROWAVE STUDIO simulation. (a) Field vectors around the DRs. (b) Relative field strength along the $z$-axis. The slight asymmetry of the field strength from top to bottom was due to an asymmetry in the geometry of our Rexolite sample mount. The kink at the bottom of the plot is a numerical artifact due to the complicated structure being modeled.

We swept the frequencies and measured the reflected power with an Agilent 773D directional coupler and an Agilent 8474B microwave detector. The reflected power decreased to zero when the microwave source hit the cavity's resonant frequency.

In addition to the resonant frequency, the $Q$-factor of a given cavity configuration can be obtained by measuring the width of the absorption resonance, $Q=f_{\text {res }} / \Delta f$. The experimentally measured resonant frequencies and $Q$-factors are given in Table I; the resonant frequencies are plotted in Fig. 4.

\section{B. ESR experiments}

To verify that the cavity was indeed supplying an oscillating magnetic field of the right character at the position of the sample, we performed optically detected magnetic

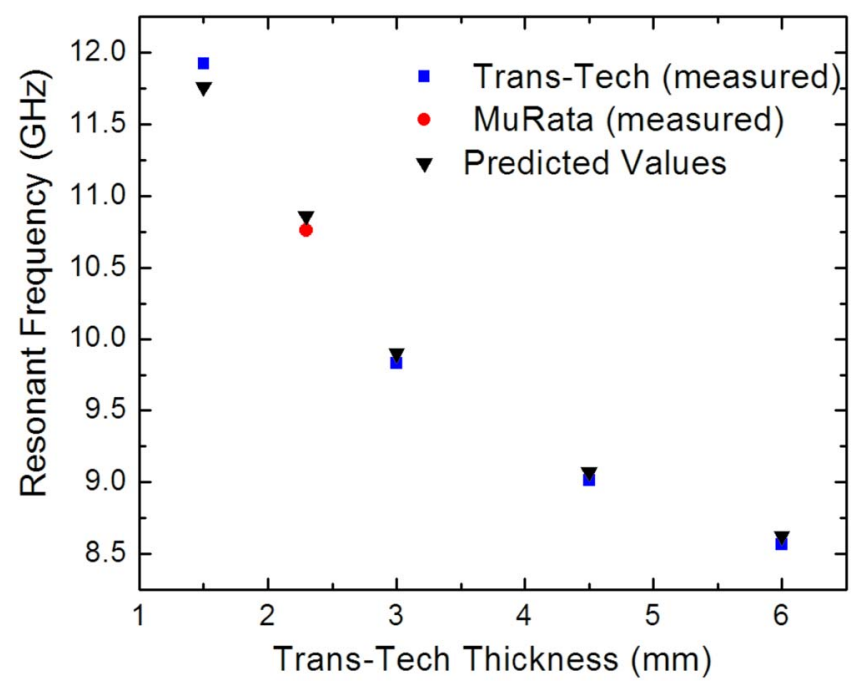

FIG. 4. (Color online) The resonant frequencies obtained for different thicknesses of the Trans-Tech DRs. In order to plot the Murata DR on the same graph, we calculated an "equivalent Trans-Tech thickness" of $2.3 \mathrm{~mm}$ for it-the thickness a Trans-Tech DR would need to be to yield the Murata DR's resonant frequency. (The actual Murata thickness is $2.44 \mathrm{~mm}$ ). 


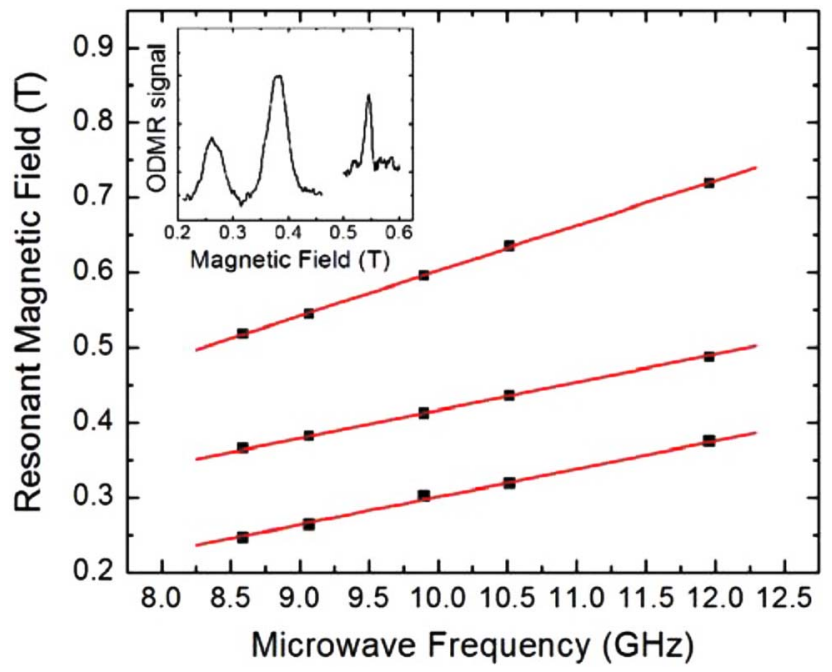

FIG. 5. (Color online) ODMR of InP:Zn at $1.5 \mathrm{~K}$. Inset: the three ESR peaks resonated by $9.065 \mathrm{GHz}$ microwaves, $25 \mathrm{dBm}$. Main figure: the ESR peak positions as a function of microwave frequency for five different cavity configurations.

resonance (ODMR) on a zinc-doped InP semiconductor. This material is well-known and the sample we used had been previously studied using ODMR by Kennedy and Glaser. ${ }^{11}$ The InP contains two defects that can be probed by ODMR: a shallow $\mathrm{Zn}$ donor with a $g$-factor of 1.20 and a deep $P_{\text {In }}$ antisite defect with $g$-factor of 2.00. The latter defect's signal is split into two lines because of hyperfine interaction with the $100 \%$ abundant ${ }^{31} \mathrm{P}$ nucleus, with a splitting of $104.2 \mathrm{mT}$. The defect density is about $2 \times 10^{15}$ defects $/ \mathrm{cm}^{3}$.

We excited luminescence of the InP sample with a $5 \mathrm{~mW}$ focused HeNe laser beam at $633 \mathrm{~nm}$ and used a long-pass filter at our detector to separate the photoluminescence (PL) from the laser light. The spin resonance was observed via a change in PL intensity. In a typical experiment, we used a PIN diode to chop the microwave signal at $125 \mathrm{~Hz}$ and a lock-in amplifier to detect the signal coming from our optical detector at that frequency. We clearly observed the three characteristic peaks with signal/noise ratio as good as that in the literature (see Fig. 5 inset). The ESR peak positions of course depend on the microwave frequency, which in turn depends on the cavity configuration. By changing the DR configuration inside the cavity, we obtained peak positions for the five different resonant frequencies (see Fig. 5). The $g$-factor of each transition can be obtained through Eq. (1) from the slope of $B_{\text {res }}$ versus $f_{\text {res. }}$. For the two peaks at lower fields, the $g$-factor obtained this way is near 2; for the peak at the higher field, the $g$-factor is 1.2. These values agree with the values measured by Kennedy and Glaser. ${ }^{11}$ Lastly, from the details of the optical setup and the number of defects in the sample, we estimate the sensitivity of these particular experiments to be approximately $2 \times 10^{5}$ electron spins, using a $1 \mathrm{~s}$ time constant. We could likely have made the experiments much more sensitive by, e.g., increasing our time constant or by taking additional steps to fully optimize the laser power density at the sample.

\section{NMR experiments}

To test the NMR coils, we performed optically detected NMR (ODNMR) on an $n$-type GaAs sample, doped at 1

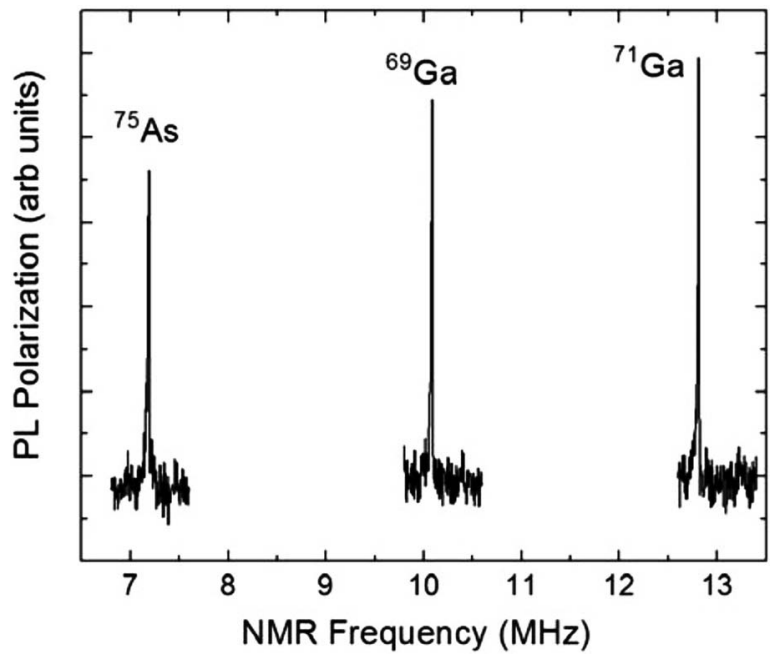

FIG. 6. NMR of $n$-GaAs at a nominal $1 \mathrm{~T}$, detected through the polarization of the PL emission. The rf was applied via the pseudo-Helmholtz coil.

$\times 10^{15} \mathrm{~cm}^{-3}$. We shined a $781 \mathrm{~nm}$ circular polarized laser beam at the sample, which oriented the electronic and nuclear spins. rf was applied to the sample via the pseudoHelmholtz coil described above. We swept the rf through the nuclear resonant frequencies and monitored the degree of circular polarization of the emitted PL. When the nuclei hit their resonant frequency, the nuclear polarization changes, which then affects the electronic polarization (due to the Overhauser effect) and hence the degree of polarization of the emitted PL. This is similar to the technique used in the ODNMR experiment performed by Brown et al. ${ }^{32}$ The ODNMR signal at $1 \mathrm{~T}$ can be seen in Fig. 6. Since we are detecting signal via the polarization of the localized donor electrons, ${ }^{33}$ we can estimate the sensitivity by estimating how many nuclei interact with all of the donor electrons within our optical beam. The sensitivity thus estimated (given a $1 \mathrm{~s}$ time constant) was $1 \times 10^{12}$ nuclear spins.

\section{CONCLUSION}

In conclusion, we constructed a microwave resonant cavity with the following notable features: (1) selectable resonant frequencies ranging from $8.5-12 \mathrm{GHz},(2)$ a diameter of $0.75 \mathrm{in}$. to fit inside a $1 \mathrm{in}$. magnet bore, (3) optical access for ODMR experiments, and (4) a coil to provide frequencies for simultaneous NMR. We note that an even greater frequency range is possible with a larger selection of interchangeable dielectrics. We tested the cavity and found it to perform well in all areas: the resonant frequencies match simulations, the quality is high, and the characterizing optically detected ESR and NMR experiments were successful. This cavity should be well suited for optically detected spinecho-type experiments on GaAs and related materials.

\section{ACKNOWLEDGMENTS}

We thank Andrzej Sienkiewicz for useful discussions regarding using DRs in microwave cavities, and for giving us the two Murata resonators. We also thank Tom Kennedy for useful discussions involving microwave cavities with optical access. We gratefully acknowledge the financial support of 
the National Science Foundation, Research Corporation, and the American Chemical Society Petroleum Research Fund. Finally, we thank CST for the free use of their Microwave Studio program.

${ }^{1}$ Semiconductor Spintronics and Quantum Computation, edited by D. D. Awschalom, D. Loss, and N. Samarth (Springer, Berlin, 2002).

${ }^{2}$ R. S. Alger, Electron Paramagnetic Resonance: Techniques and Applications (Interscience, New York, 1968).

${ }^{3}$ E. L. Hahn, Phys. Rev. 80, 580 (1950).

${ }^{4}$ For some of the groundbreaking results which showed long spin lifetimes in GaAs, see, e.g., J. M. Kikkawa and D. D. Awschalom, Phys. Rev. Lett. 80, 4313 (1998); R. I. Dzhioev, K. V. Kavokin, V. L. Korenev, M. V. Lazarev, B. Ya. Meltser, M. N. Stepanova, B. P. Zakharchenya, D. Gammon, and D. S. Katzer, Phys. Rev. B 66, 245204 (2002).

${ }^{5}$ C. Weisbuch and C. Hermann, Phys. Rev. B 15, 816 (1977).

${ }^{6}$ J. S. Colton, T. A. Kennedy, A. S. Bracker, and D. Gammon, Phys. Rev. B 69, 121307 (2004).

${ }^{7}$ J. S. Colton, M. E. Heeb, P. Schroeder, A. Stokes, L. R. Wienkes, and A. S. Bracker, Phys. Rev. B 75, 205201 (2007).

${ }^{8}$ K-M. C. Fu, W. Yeo, S. Clark, C. Santori, C. Stanley, M. C. Holland, and Y. Yamamoto, Phys. Rev. B 74, 121304(R) (2006).

${ }^{9}$ V. N. Golovach, A. Khaetskii, and D. Loss, Phys. Rev. Lett. 93, 016601 (2004).

${ }^{10}$ M. J. Snelling, G. P. Flinn, A. S. Plaut, R. T. Harley, A. C. Tropper, R. Eccleston, and C. C. Phillips, Phys. Rev. B 44, 11345 (1991).

${ }^{11}$ T. A. Kennedy and E. R. Glaser, in Identification of Defects in Semiconductors, Semiconductors and Semimetals Vol. 51A, edited by Michael Stavola (Academic, New York, 1998), Chap. 3.

${ }^{12}$ R. Hanson, O. Gywat, and D. D. Awschalom, Phys. Rev. B 74, 161203 (2006).

${ }^{13}$ Optical Orientation, edited by F. Meier and B. P. Zakharchenya (NorthHolland, Amsterdam, 1984).

${ }^{14}$ See, e.g., the paper by Kikkawa and Awschalom mentioned in Ref. 4; there has been much subsequent time-resolved Faraday/Kerr rotation work by that group and others.
${ }^{15}$ See, e.g., Ref. 2, p. 127.

${ }^{16}$ J. S. Colton, T. A. Kennedy, A. S. Bracker, D. Gammon, and J. B. Miller, Phys. Rev. B 67, 165315 (2003).

${ }^{17}$ J. S. Colton, T. A. Kennedy, A. S. Bracker, J. B. Miller, and D. Gammon, Solid State Commun. 132, 613 (2004).

${ }^{18}$ D. Richtmyer, J. Appl. Phys. 10, 391 (1939).

${ }^{19}$ G. Annino, M. Cassettari, I. Longo, and M. Martinelli, Appl. Magn. Reson. 16, 45 (1999).

${ }^{20}$ A. Sienkiewicz, K. Qu, and C. P. Scholes, Rev. Sci. Instrum. 65, 68 (1994).

${ }^{21}$ A. Sienkiewicz, B. Vileno, S. Garaj, M. Jaworski, and L. Forro, J. Magn. Reson. 177, 261 (2005).

${ }^{22}$ M. Schreiner, "Optisch detektierte magnetische Kernspin-Elektronenspin Doppelresonanz in AlGaAs/GaAs Heterostrukturen," Ph.D. thesis, Universität Bayreuth, Bayreuth, Germany, 1995.

${ }^{23}$ M. Schreiner, H. Pascher, G. Denninger, S. A. Studenikin, G. Weimann, and R. Lösch, Solid State Commun. 102, 715 (1997).

${ }^{24}$ E. Stavitski, L. Wagnert, and H. Levanon, J. Phys. Chem. A 109, 976 (2005).

${ }^{25}$ A. Blank, E. Stavitski, H. Levanon, and F. Gubaydullon, Rev. Sci. Instrum. 74, 2853 (2003).

${ }^{26}$ A. Sienkiewicz, B. G. Smith, A. Veselov, and C. P. Scholes, Rev. Sci. Instrum. 67, 2134 (1996).

${ }^{27}$ H. Seidel, Z. Phys. 165, 239 (1961).

${ }^{28}$ K. P. Dinse, I. Möbius, and R. Biehl, Z. Naturforsch. B 28, 1069 (1973).

${ }^{29}$ See, e.g., J. D. Jackson, Classical Electrodynamics, 3rd ed. (Wiley, New York, 1998).

${ }^{30}$ D. Kaifez and P. Guillon, Dielectric Resonators, 2nd ed. (Noble, Atlanta, 1998).

${ }^{31}$ M. Jaworski, A. Sienkiewicz, and C. P. Scholes, J. Magn. Reson. 124, 87 (1997).

${ }^{32}$ S. W. Brown, T. A. Kennedy, E. R. Glaser, and D. S. Katzer, J. Phys. D 30, 1411 (1997).

${ }^{33}$ The electron spin polarization measured via polarization of PL from photoexcited electrons reflects the spin of the overall doped electron reservoir; see, e.g., Ref. 16. 